\title{
Estratégias tecnológicas utilizadas no ensino durante a pandemia
}

\section{Technological strategies used in teaching during the pandemic \\ Estrategias tecnológicas utilizadas en la enseñanza durante la pandemia}

Socorro de Maria Rodrigues Sousa ORCID: https://orcid.org/0000-0003-3488-7757 Faculdade IESM, Brasil

E-mail: s16maria@hotmail.com Gabriela Oliveira Parentes da Costa ORCID: https://orcid.org/0000-0001-9473-8986 Instituto Federal do Maranhão, Brasil E-mail: gabiparents@hotmail.com Rogério Pinto de Sousa ORCID: https://orcid.org/0000-0002-1619-938X Instituto Federal de Roraima, Brasil E-mail: rogeriopinto040@gmail.com

Micilane Nascimento dos Santos ORCID: https://orcid.org/0000-0002-0397-2441 Instituto Federal do Maranhão, Brasil E-mail: micysantos15@gmail.com Naianne Geórgia Sousa de Oliveira ORCID: https://orcid.org/0000-0002-2949-0803 Faculdade Santa Luzia, Brasil

E-mail: naianne.enfermeira@gmail.com

Luciana Spindola Monteiro Toussaint ORCID: https://orcid.org/0000-0001-7691-1570 Fundação Municipal de Saúde, Brasil E-mail: lucianaspindola09@gmail.com

Sarah Carolina Borges Mariano ORCID: https://orcid.org/0000-0003-0452-0516 Universidade Federal do Piauí, Brasil E-mail: sarahcborges14@gmail.com

Verônica Maria de Sena Rosal ORCID: https://orcid.org/0000-0001-7349-9955 Universidade Federal do Piauí, Brasil E-mail: veronica.rosal@hotmail.com

Ravena de Sousa Alencar Ferreira ORCID: https://orcid.org/0000-0001-7311-2212 Universidade Federal do Piauí, Brasil E-mail: ravenaa89@gmail.com Laíse Virgínia Soares Senna ORCID: https://orcid.org/0000-0003-0144-7763 Universidade Federal do Piauí, Brasil E-mail: laisesenna@gmail.com

Érida Zoé Lustosa Furtado ORCID: https://orcid.org/0000-0002-6162-7558 Universidade Federal do Piauí, Brasil E-mail: eridazoe@hotmail.com

Lilian Ferreira do Nascimento ORCID: https://orcid.org/0000-0003-3725-3872 Universidade Federal do Piauí, Brasil

E-mail:liliannascimentopsi@gmail.com

Verônica Elis Araújo Rezende ORCID: https://orcid.org/0000-0001-9076-3375 Universidade Federal do Piauí, Brasil

E-mail: veronicaelisrezende@yahoo.com.br

Otília Maria Reis Sousa Tinel

ORCID: https://orcid.org/0000-0001-9306-7543 Universidade Federal do Piauí, Brasil E-mail: otiliatinel1 @ outlook.com

Rebeca Natacha Barbosa Vieira ORCID: https://orcid.org/0000-0001-8306-5771 Faculdade Aliança, Brasil E-mail: rebeca.natacha02@gmail.com 


\author{
Felipe de Sousa Moreiras \\ ORCID: https://orcid.org/0000-0002-8703-1429 \\ Universidade Federal do Piauí, Brasil \\ E-mail: felipe_moreiras007@ hotmail.com
}

\begin{abstract}
Resumo
Introdução: Existem recursos tecnológicos que podem contribuir com o desenvolvimento dos discentes, assim, tem-se como objetivo: Realizar um estudo bibliográfico para conhecer quais as estratégias tecnológicas foram mais utilizadas para dar continuidade ao ensino durante a pandemia. Metodologia: Trata-se de uma pesquisa de revisão bibliográfica. O levantamento dos dados ocorreu em julho de 2021, utilizando-se os termos: Google Meet; Covid-19; WhatsApp; Mídias Digitais no Ensino. Após a etapa de refinamento, restaram 14 artigos que fizeram parte da amostra. Resultados e discussão: O Google Meet que foi a plataforma mais utilizada. Foram usados o Facebook WhatsApp como ferramentas de comunicação entre os professores e alunos. Além do Google Classroom e Gravação de Podcast. Uma estratégia que chamou a atenção foi o uso do Instagram para lives, cuja ferramenta permite que as aulas sejam salvas no IGTV da rede social, podendo ser vista quantas vezes o aluno desejar. Esta mídia social possibilita aos seus usuários diferentes experiências promovendo o engajamento e a prática educativa colaborativa. Considerações Finais: As ferramentas do Google foram as mais utilizadas, ou por serem de fácil acesso ou devido ao acesso gratuito. Percebeu-se que as redes sociais também foram exploradas para ministração das aulas. O Youtube também serviu ao mesmo propósito. O WhatsApp, além de ferramenta usada para comunicação entre os docentes e alunos também foi usado como recurso nas aulas.
\end{abstract}

Palavras-chave: Covid-19; Tecnologia educacional; Ferramentas de ensino; Mídias Digitais.

\begin{abstract}
Introduction: There are technological resources that can contribute to the development of students, thus, the aim is: Conduct a bibliographic study to know which technological strategies were most used to continue teaching during the pandemic. Methodology: This is a literature review research. Data collection took place in July 2021, using the terms: Google Meet; Covid-19; Whatsapp; Digital Media in Teaching. After the refinement step, 14 articles remained that were part of the sample. Results and discussion: Google Meet which was the most used platform. Facebook WhatsApp was used as communication tools between teachers and students. Plus Google Classroom and Podcast Recording. One strategy that drew attention was the use of Instagram for Lives, whose tool allows classes to be saved on the social network's IGTV, and can be viewed as many times as the student wants. This social media enables its users to have different experiences promoting engagement and collaborative educational practice. Final Remarks: Google tools were the most used, either because they are easy to access or because of free access. It was noticed that social networks were also exploited for teaching classes. Youtube also served the same purpose. WhatsApp, in addition to being a tool used for communication between teachers and students, was also used as a resource in classes. Keywords: Covid-19; Educational technology; Teaching tools; Digital Media.
\end{abstract}

\begin{abstract}
Resumen
Introducción: Existen recursos tecnológicos que pueden contribuir al desarrollo de los estudiantes, por lo que el objetivo es: Realizar un estudio bibliográfico para conocer qué estrategias tecnológicas fueron más utilizadas para continuar la docencia durante la pandemia. Metodología: Se trata de una investigación de revisión de la literatura. La recopilación de datos se llevó a cabo en julio de 2021, utilizando los términos: Google Meet; COVID-19; Whatsapp; Medios digitales en la docencia. Tras el paso de refinamiento, quedaron 14 artículos que formaban parte de la muestra. Resultados y discusión: Google Meet que fue la plataforma más utilizada. Se utilizó Facebook WhatsApp como herramienta de comunicación entre profesores y alumnos. Además, Google Classroom y grabación de podcasts. Una estrategia que llamó la atención fue el uso de Instagram for Lives, cuya herramienta permite que las clases se guarden en el IGTV de la red social y se puedan visualizar tantas veces como el alumno desee. Esta red social permite a sus usuarios tener diferentes experiencias promoviendo el compromiso y la práctica educativa colaborativa. Observaciones finales: Las herramientas de Google fueron las más utilizadas, ya sea por su fácil acceso o por su acceso gratuito. Se notó que las redes sociales también fueron explotadas para impartir clases. Youtube también cumplió el mismo propósito. WhatsApp, además de ser una herramienta de comunicación entre profesores y alumnos, también se utilizó como recurso en las clases.
\end{abstract}

Palabras clave: COVID-19; Tecnologia educacional; Herramientas de enseñanza; Medios digitales.

\title{
1. Introdução
}

Em consequência da pandemia causada pelo Coronavírus, em 2020 o Ministério da Saúde (MS) publicou em Boletim Epidemiológico a necessidade do distanciamento social. Logo após, o Ministério da Educação (MEC) emitiu uma Portaria orientando sobre a urgência da paralisação das aulas presenciais para evitar a propagação do vírus e contágios em locais 
públicos e de grande aglomeração. Atendendo as exigências, as atividades presenciais foram suspensas, imaginando-se que seria por pouco tempo, contudo, percebendo-se a impossibilidade do retorno às escolas, houve a necessidade de se adaptar, buscando estratégias para que as aulas não continuassem paradas (Carneiro et al., 2020).

A partir desses desafios, o uso das tecnologias na educação ganhou força em todo o mundo, mostrando as possibilidades de ensino que o ambiente virtual é capaz de proporcionar (Sousa et al., 2021).

As tecnologias na educação favorecem a difusão do conhecimento e contribuem para o compartilhamento de informações entre os conectados, seja professor ou aluno, e este tem a possibilidade de acessar as informações com apenas um clique, facilitando a difusão de novos conhecimentos (Carneiro et al., 2020).

Para Kenski (2007), as tecnologias auxiliam no processo educacional, uma vez que estão presentes em vários momentos do planejamento pedagógico e contribuem para a organização do ensino.

Questões sobre as ferramentas digitais vêm sendo mais discutidas desde o início da pandemia da COVID-19 e vem sendo considerada como uma forma de democratização do ensino, facilitando que a educação chegue em muitos lugares, apesar de não ser de forma justa e igualitária para todos (Santos, 2020).

As escolas se adequaram ao novo formato exigido devido às circunstâncias, deixando um pouco o uso do quadro e giz, pincel, ou projetor de slides, e aderindo à ferramentas tecnológicas como suporte educacional (Valente et al., 2020). Para isso, houve a necessidade de oferta de cursos para todos os envolvidos nesse processo, principalmente, os professores (Da Silva, 2021).

Dentre os diversos recursos digitais e tecnológicos utilizados nas aulas remotas durante a pandemia, podem ser citadas o Google, o Google Classrom, o Google meet, o Google Suite, Google Drive, o Zoom, o Skype, o Moodle, o Microsoft Teams, o Onenote from Microsoft, Seesaw, ManageBac, ManageBac, Ed Dojo EdModo, Mediawijs, entre outras e até as mídias sociais como o YouTube, Instagram e WhatsApp. Tais ferramentas permitiram o acesso remoto às atividades (Moreira et al., 2020).

Existem muitos recursos tecnológicos que podem contribuir com o desenvolvimento de habilidades e competências dos discentes, assim, tem-se como objetivo: Realizar um estudo bibliográfico para conhecer quais as estratégias tecnológicas foram mais utilizadas para dar continuidade ao ensino durante a pandemia.

A inquietação em pesquisar sobre a temática surgiu após uma atividade acadêmica dos autores, que consistia em realizar perguntas aos docentes sobre o uso das tecnologias em educação. Em um dos questionamentos, os professores relataram quais as ferramentas digitais mais utilizadas durante a pandemia, sendo elas o próprio Google, o canva, vídeos do YouTube, Mentimenter, flipty, Google drive, jamboatd, Plataforma Zoom, Google Meet, e Microsoft Teams, Kahoot, Socrative, Power point, Padlet, Adobe Spark, WhatsApp, Liveworksheets, Padlet, Socrative, Google Forms, Google Classroom e o Adobe Spark.

\section{Metodologia}

Trata-se de uma pesquisa de revisão bibliográfica, cujo foco está na resolução de um problema ou hipótese através de referenciais teóricos já publicados. A partir desse levantamento, é realizada uma análise e discussão dos achados, subsidiando conhecimento acerca do tema, de forma sistemática, desde o início da definição temática até a divulgação dos achados (De Sousa et al., 2021).

O levantamento dos dados ocorreu em julho de 2021, utilizando-se os termos: Google Meet; Covid-19; WhatsApp; Mídias Digitais no Ensino. Encontrou-se 1.890 resultados no Google acadêmico. Após a etapa de refinamento, restaram 14 artigos que fizeram parte da amostra. 
As etapas seguidas para o desenvolvimento desta bibliografia foram as orientadas e descritas por Gil (2002); Lakatos \& Marconi (2003) conforme Figura 1.

Figura 1: Etapas da pesquisa Bibliográfica.

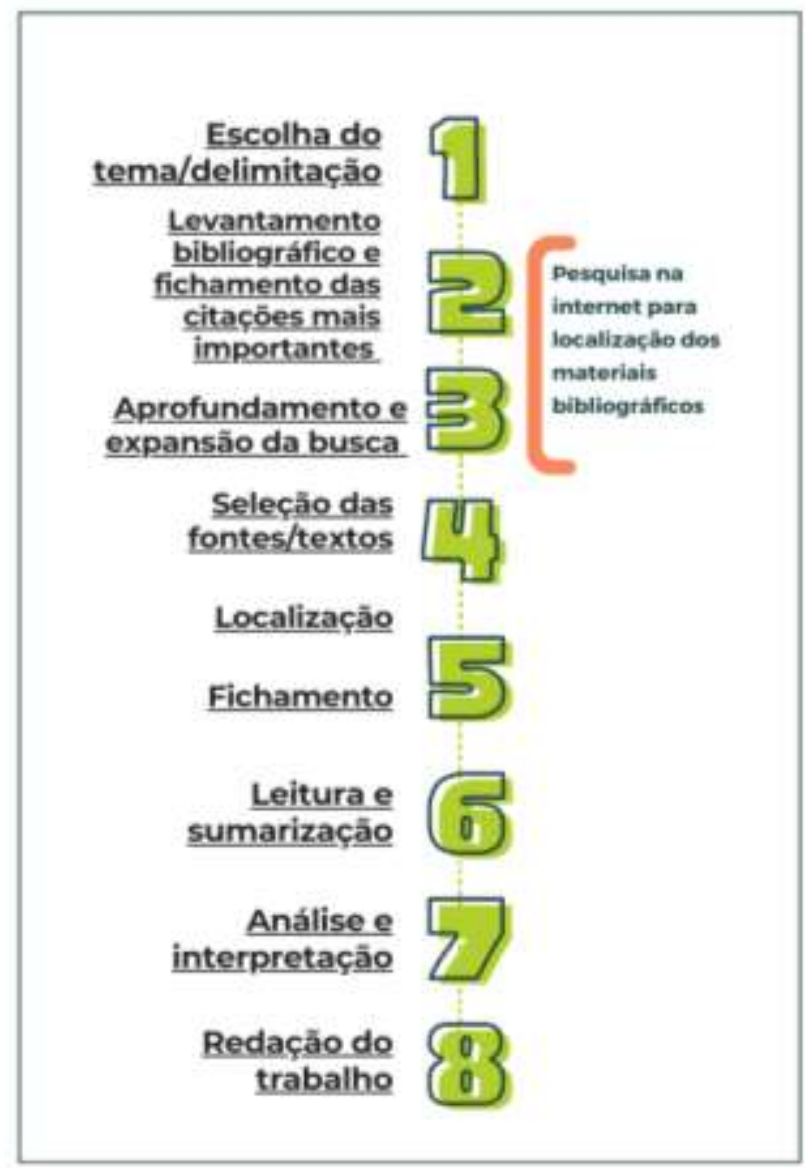

Fonte: Adaptado de Gil (2002); Lakatos e Marconi (2003).

Os critérios de inclusão foram materiais primários sobre as tecnologias ou ferramentas digitais utilizadas no ensino durante a pandemia, publicadas entre 2020 até junho de 2021, em qualquer idioma. Os critérios de exclusão foram as bibliografias que tratam sobre tecnologias, mas não citaram especificamente nenhuma das ferramentas digitais utilizadas, materiais publicados em anais de congressos, teses e revisões.

Os dados coletados foram analisados por meio da Análise de Conteúdo desenvolvida por Bardin (2010) e discutidos de forma descritiva.

\section{Resultados e Discussão}

Dos 1.890 artigos encontrados, após a leitura dos títulos e resumos, restaram 149 que após a leitura na íntegra, 135 deles foram descartados, restando 14 artigos que fizeram parte dessa pesquisa (Quadro 1). 
Quadro 1: Informações da amostra: Estratégias tecnológicas utilizadas no ensino durante a pandemia. Brasil, 2021.

\begin{tabular}{|c|c|c|c|c|c|c|}
\hline Título & Autores & Revista & $\begin{array}{c}\text { Amostra/ } \\
\text { Participantes } \\
\end{array}$ & $\begin{array}{c}\text { Tipo(s) de ferramenta(s) } \\
\text { aborda } \\
\end{array}$ & $\begin{array}{c}\begin{array}{c}\text { Local/Região da } \\
\text { pesquisa }\end{array} \\
\end{array}$ & Ano \\
\hline $\begin{array}{c}\text { Professores, tecnologias } \\
\text { educativas e COVID-19: } \\
\text { realidades e desafios em } \\
\text { Angola }\end{array}$ & Julião, A. L. & $\begin{array}{c}\text { Revista } \\
\text { Angolana de } \\
\text { Ciências }\end{array}$ & Professores & $\begin{array}{c}\text { Vídeo-aulas, Google } \\
\text { Classrom, vídeo-conferência, } \\
\text { Facebook, Zoom, WhatsApp }\end{array}$ & $\begin{array}{l}8 \text { províncias de Angola: } \\
\text { Benguela, Huíla, } \\
\text { Huambo, Luanda, } \\
\text { Namibe, Bengo, Bié e } \\
\text { Moxico }\end{array}$ & 2020 \\
\hline $\begin{array}{c}\text { Estratégias e desafios da } \\
\text { atuação docente de uma } \\
\text { professora no contexto da } \\
\text { pandemia da Covid- } 19 .\end{array}$ & $\begin{array}{l}\text { Scalabrin, A. } \\
\text { M. M. O. \& } \\
\text { Mussato, S }\end{array}$ & $\begin{array}{c}\text { Revista de } \\
\text { Educação } \\
\text { Matemática }\end{array}$ & $\begin{array}{l}\text { Professores e } \\
\text { alunos }\end{array}$ & $\begin{array}{c}\text { Videoaulas, YouTube Google } \\
\text { Forms, Google Classroom, } \\
\text { WhatsApp }\end{array}$ & Boa Vista-RR & 2020 \\
\hline $\begin{array}{l}\text { O uso das Plataformas } \\
\text { Digitais pelas IES no } \\
\text { contexto de afastamento } \\
\text { social pela Covid-19. }\end{array}$ & $\begin{array}{l}\text { Góes, C. B, \& } \\
\text { Cassiano, G. }\end{array}$ & $\begin{array}{c}\text { Folha de } \\
\text { rosto: Revista } \\
\text { de } \\
\text { Bibliotecono } \\
\text { mia e Ciência } \\
\text { da } \\
\text { Informação. }\end{array}$ & Professores & $\begin{array}{c}\text { Google Meet, Zoom, Skype, } \\
\text { Google Meet }\end{array}$ & $\begin{array}{c}\text { Feira de Santana-BA e } \\
\text { Cachoeira-BA }\end{array}$ & 2020 \\
\hline $\begin{array}{l}\text { O uso de tecnologias digitais } \\
\text { e mídias sociais por } \\
\text { profissionais da saúde no } \\
\text { período da pandemia da } \\
\text { COVID-19. }\end{array}$ & $\begin{array}{l}\text { Bueno, M. B. } \\
\text { T.; Bueno, M. } \\
\text { M.; Moreira } \\
\text { \& M. I. G. }\end{array}$ & $\begin{array}{l}\text { Revista } \\
\text { Thema }\end{array}$ & $\begin{array}{l}\text { Profissionais } \\
\text { da área da } \\
\text { saúde } \\
\text { (Fisioterapeut } \\
\text { as, Educador } \\
\text { Físico, } \\
\text { Psicólogo, } \\
\text { Médica } \\
\text { Veterinária } \\
\text { Médico e } \\
\text { Enfermeiro }\end{array}$ & $\begin{array}{c}\text { WhatsApp, Instagram, zoom, } \\
\text { Telegram, Twitter, Linkedin, } \\
\text { Whereby e o Hangouts }\end{array}$ & $\begin{array}{c}\text { Regiões Sul e Sudeste do } \\
\text { Brasil }\end{array}$ & 2021 \\
\hline $\begin{array}{l}\text { Construção de tecnologias } \\
\text { educativas como forma de } \\
\text { educação em saúde para a } \\
\text { prevenção da Covid-19: } \\
\text { relato de experiência. }\end{array}$ & $\begin{array}{l}\text { Batista Neto, } \\
\text { J. B. S. et al. }\end{array}$ & $\begin{array}{l}\text { Revista } \\
\text { Eletrônica } \\
\text { Acervo } \\
\text { Saúde. }\end{array}$ & $\begin{array}{l}\text { Enfermeira- } \\
\text { docente e } \\
\text { acadêmicos } \\
\text { de } \\
\text { enfermagem }\end{array}$ & $\begin{array}{l}\text { Power Point, Powtoon, } \\
\text { WhatsApp, Facebook e } \\
\text { Instagram }\end{array}$ & $\begin{array}{l}\text { Seis municípios da } \\
\text { região do lago de } \\
\text { Tucuruí, no interior do } \\
\text { Pará. }\end{array}$ & 2020 \\
\hline $\begin{array}{l}\text { As tecnologias digitais no } \\
\text { processo de ensino e } \\
\text { aprendizagem de línguas } \\
\text { estrangeiras - estudo de caso } \\
\text { de escola da Rede Federal de } \\
\text { Ensino - CE/Brasil - em } \\
\text { contexto anterior ao da } \\
\text { pandemia de covid-19. }\end{array}$ & $\begin{array}{l}\text { Monteiro, F. } \\
\text { N. S. P. }\end{array}$ & $\begin{array}{c}\text { Research, } \\
\text { Society and } \\
\text { Development. }\end{array}$ & Professores & $\begin{array}{l}\text { WhatsApp, Linkedin, Google } \\
\text { Maps, Google tradutor, } \\
\text { Kahoot e Quizlet, Netflix, } \\
\text { Longman English Dicionary, } \\
\text { VOA learning english, entre } \\
\text { outros }\end{array}$ & Ceará & 2020 \\
\hline $\begin{array}{c}\text { A utilização da mídia } \\
\text { podcast como prática } \\
\text { inovadora na educação } \\
\text { superior. }\end{array}$ & $\begin{array}{l}\text { De Paula, S. } \\
\text { A. \& } \\
\text { Figueiró, R. }\end{array}$ & $\begin{array}{l}\text { Research, Soci } \\
\text { ety and } \\
\text { Development }\end{array}$ & $\begin{array}{l}\text { Professores e } \\
\text { alunos }\end{array}$ & Podcast & Volta Redonda-RJ & 2020 \\
\hline $\begin{array}{l}\text { Formação de professores da } \\
\text { Educação Básica para uso } \\
\text { das ferramentas Google na } \\
\text { educação: uma experiência } \\
\text { extensionista em tempos de } \\
\text { pandemia. }\end{array}$ & $\begin{array}{l}\text { Dias, C. R. S. } \\
\text { D. et al. }\end{array}$ & $\begin{array}{c}\text { IX Congresso } \\
\text { Brasileiro de } \\
\text { Informática } \\
\text { na Educação } \\
\text { (CBIE 2020). } \\
\text { Anais do } \\
\text { XXVI } \\
\text { Workshop de } \\
\text { Informática } \\
\text { na Escola } \\
\text { (WIE 2020). }\end{array}$ & $\begin{array}{l}\text { Professores e } \\
\text { alunos }\end{array}$ & $\begin{array}{c}\text { Google for Education: } \\
\text { Google Classroom, Meet } \\
\text { Hangout, Google Drive e } \\
\text { Google Formulários }\end{array}$ & Pará & 2020 \\
\hline $\begin{array}{c}\text { AVAS21 - Ambiente virtual } \\
\text { de aprendizagem para o } \\
\text { século 21: uma experiência } \\
\text { exitosa. }\end{array}$ & $\begin{array}{l}\text { Torres, R. M. } \\
\text { et al. }\end{array}$ & $\begin{array}{c}\text { Brazilian } \\
\text { Journal of } \\
\text { Development. }\end{array}$ & $\begin{array}{l}\text { Professores e } \\
\text { alunos }\end{array}$ & Podcast & Minas Gerais & 2021 \\
\hline $\begin{array}{c}\text { Impactos da pandemia } \\
\text { covid-19 nas aulas de inglês. }\end{array}$ & $\begin{array}{l}\text { Denardi, D. } \\
\text { A. C.; } \\
\text { Amoroginski } \\
\text { Marcos, R. \& } \\
\text { Stankoski, C. }\end{array}$ & $\begin{array}{c}\text { Ilha do } \\
\text { Desterro. }\end{array}$ & Professores & $\begin{array}{l}\text { Kahoot e Google Maps, } \\
\text { Google Meet, Zoom, Bongo, } \\
\text { Skype, Google Hangouts, } \\
\text { Microsoft Teams, WhatsApp, } \\
\text { Google Classroom, }\end{array}$ & $\begin{array}{c}\text { Região sudoeste do } \\
\text { Paraná e oeste de Santa } \\
\text { Catarina }\end{array}$ & 2021 \\
\hline
\end{tabular}




\begin{tabular}{|c|c|c|c|c|c|c|}
\hline & $\mathrm{R}$. & & & Screencastify, YouCute & & \\
\hline $\begin{array}{l}\text { O Ensino da Anamnese } \\
\text { Assistido por Tecnologias } \\
\text { Digitais durante a Pandemia } \\
\text { da Covid-19 no Brasil. }\end{array}$ & $\begin{array}{l}\text { Magalhães, } \\
\text { A. J. A. et al. }\end{array}$ & $\begin{array}{l}\text { Revista } \\
\text { brasileira de } \\
\text { educação } \\
\text { médica. } \\
\end{array}$ & $\begin{array}{l}\text { Alunos do } \\
\text { curso de } \\
\text { medicina }\end{array}$ & $\begin{array}{l}\text { Podcast, padlet, Anchor, } \\
\text { Spotify, Kahoot }\end{array}$ & Arapiraca- Alagoas & 2020 \\
\hline $\begin{array}{l}\text { As estratégias dos docentes } \\
\text { com o uso de tecnologias } \\
\text { digitais no contexto } \\
\text { pandêmico da covid-19. }\end{array}$ & $\begin{array}{l}\text { Gomes, E. G. } \\
\text { S. \& } \\
\text { Carvalho, A. } \\
\text { B. G. P. }\end{array}$ & $\begin{array}{c}\text { EM TEIA- } \\
\text { Revista de } \\
\text { Educação } \\
\text { Matemática e } \\
\text { Tecnológica } \\
\text { Iberoamerica } \\
\text { na. } \\
\end{array}$ & & $\begin{array}{c}\text { Google Meet, Padlet, mapa } \\
\text { mental, Mentimeter, Kahoot, } \\
\text { Google Forms, Zoom, } \\
\text { Flashback Express, Vidyard, } \\
\text { Google Classroom, YouTube }\end{array}$ & Pernambuco & 2020 \\
\hline $\begin{array}{c}\text { Tecnologias digitais e } \\
\text { educação escolar em tempos } \\
\text { de pandemia da Covid-19: } \\
\text { Percepções de professores/as } \\
\text { de Língua Portuguesa. }\end{array}$ & $\begin{array}{l}\text { Santos, A. C.; } \\
\text { Santos, N. A. } \\
\text { \& Santos, W. } \\
\text { P. }\end{array}$ & $\begin{array}{l}\text { Educação, } \\
\text { Sociedade \& } \\
\text { Culturas. }\end{array}$ & & $\begin{array}{l}\text { Facebook, WhatsApp, Google } \\
\text { Classroom, Google Meet, } \\
\text { Socrative, Google forms, } \\
\text { Podcast, Instagram }\end{array}$ & Alagoas & 2021 \\
\hline
\end{tabular}

Fonte: Autores.

De acordo com a pesquisa de Julião (2020), na Angola, 34,8\% dos professores pesquisados afirmaram que possuíam maior domínio em gravar vídeo-aulas, 1,4\% em utilizar o Google Classroom e 8,7\% em utilizar vídeo-conferência. Questionados sobre quais ferramentas mais utilizavam para comunicação com os alunos, a maior parte deles (60,9\%) afirmou não usar e 31,9\% afirmaram usar o Facebook.

Em uma pesquisa com 502 docentes, percebeu-se que 185 deles não desenvolveram atividade com mediação tecnológica durante a pandemia e alegaram a falta de formação para o uso das tecnologias como um fator consequente para isso (Nonato et al. 2021). Os recursos tecnológicos na educação são uma realidade indiscutível na era atual. Os protagonistas do ensino devem acompanhar as mudanças, sendo necessário apostar na formação tecnológica para inclusão e manuseio dessas ferramentas na escola.

Scalabrin e Mussato (2020) também utilizaram para aulas de matemática a gravação de vídeos como estratégia para a ministração de aulas. O Youtube foi outra ferramenta que as autoras mencionaram, além do uso do Google Forms para aplicação das provas e do Google Classroom para criação de uma sala virtual e grupos de WhatsApp para comunicação e interação professor e aluno.

Professores das escolas municipais de Cuiabá também utilizaram as redes sociais como o Facebook e Instagram para ministração de aulas e comunicação com os alunos, além do Google Sala de Aula, Moodle e o e-mail (Godoi et al. 2021).

Segundo Iftakhar (2016), o Google Classroom ou Google sala de aula é uma ferramenta educacional que proporciona aos professores e alunos uma relação mais interativa e comunicativa promovendo a realização de conteúdos adequados para o processo de construção do conhecimento. A plataforma é online e gratuita, os recursos disponibilizados facilitam a organização das aulas virtuais. Esta ferramenta está disponível para uso em computadores, smartphones e tablets, que pode ser acessada pelo Google (Classroom, 2020).

Outra ferramenta tecnológica bastante usada nas aulas remotas é a plataforma Moodle, que é uma sala de aula virtual que possibilita aos educadores e alunos um ambiente de aprendizagem personalizado que disponibiliza ferramentas flexíveis para facilitar o processo de ensino/aprendizagem. Na plataforma, os usuários têm acesso a fóruns, wikis, chats e blogs que são ferramentas colaborativas para o andamento das aulas virtuais (Moodle, 2021).

Góes e Cassiano (2020) evidenciaram o uso de uma variedade de ferramentas pelos professores pesquisados, além do Google Meet que foi a plataforma mais utilizada, o Zoom e o Skype, também foram citados como estratégias para dar continuidade ao ensino.

Nessa perspectiva, com o cenário pandêmico da COVID-19 houve uma necessidade de uso de plataformas digitais no contexto escolar para dar continuidade ao ensino e aprendizagem proporcionando uma relação interativa entre alunos e educadores. Dessa forma, o Google Meet se tornou uma ferramenta bastante utilizada no âmbito educacional, no entanto seu 
uso evidenciou a precariedade do sistema educacional brasileiro que não estava apto para mudanças radicais na área da educação. Com isso, docentes e alunos tiveram que se reinventar frente à nova realidade (Senhoras, 2021; Dias \& Pinto, 2020).

Outra ferramenta tecnológica muito usada na pandemia é o Zoom que é uma plataforma online de videoconferência onde o aluno ingressa na aula virtual por meio de um link enviado pelo docente (Zoom, 2020). A plataforma permite aos usuários participar de forma ativa como nas aulas presenciais, por acontecer em tempo real é possível que os alunos tirem dúvidas, entrem em debates, mas é preciso que o professor busque alternativas para fazer os discentes interagir.

$\mathrm{Na}$ disciplina de língua portuguesa, em Alagoas, também foram usados o Facebook WhatsApp como ferramentas de comunicação entre os professores e alunos. Além do Google Classroom, utilizaram o Google Meet, o Socrative em alternativa ao Google forms, Gravação de Podcast. Uma estratégia que chamou a atenção foi o uso do Instagram para lives, cuja ferramenta permite que as aulas sejam salvas no IGTV da rede social, podendo ser vista quantas vezes o aluno desejar (Santos et al., 2021).

O Instagram dispõe alguns recursos aos seus usuários como Feed, Stories e IGTV que são ferramentas imprescindíveis no processo de ensino- aprendizagem. Esta mídia social aonde prevalece o dinamismo da mensagem visual possibilita aos seus usuários diferentes experiências promovendo o engajamento desejado e como ambiente de aprendizagem possibilita uma prática educativa colaborativa e interativa (Alves et al., 2018).

Em sua pesquisa, Bueno, Bueno \& Moreira (2021) perceberam que os docentes da área da saúde fizeram uso de muitas tecnologias para ministração das aulas, sendo o WhatsApp a ferramenta mais utilizada pelos profissionais. O Instagram também foi citado pela maioria. O zoom, o Telegram foi utilizado no processo e o Twitter, o Linkedin, o Whereby e o Hangouts foram citados uma vez cada um. A estratégia de transmissão de aula ao vivo foi utilizada por meio das mídias sociais.

Outras estratégias usadas para o ensino na área da saúde, foi descrito por Batista Neto et al. (2020), em que na disciplina de "Tecnologias Educacionais em Saúde e Enfermagem" construíram materiais educativos por meio do Powtoon e WhatsApp e Instagram para divulgar o material.

Amaral e Sabota (2017) em suas pesquisas abordam o PowToon como uma ferramenta de produção de vídeo animado com boa qualidade. É disponibilizado ao usuário uma diversidade de elementos de personalização para criação do da sua animação. O PowToon pode ser inserido dentro do ambiente educacional através de oficinas, projetos, avaliações individuais ou em grupos, com qualquer faixa etária. A ferramenta digital tem a capacidade de desenvolver a autonomia dos alunos, pois o estudante ficará com o dever de elaborar e criar sua animação.

A tecnologia pode contribuir com as aulas da área da saúde, uma vez que permite melhor experiência na realização de pesquisas e criação de apresentação, além de melhorar a visualização de estruturas do corpo humano por meio de sites e aplicativos. Considerando que a formação não pode estagnar, é necessário utilizar de estratégias na área da saúde para dar continuidade ao ensino. Para tanto, é necessário que todos assumam responsabilidade na aprendizagem colaborativa utilizandose de estratégias didáticas (Salvador et al., 2019).

Os cursos da área da saúde que possuem suas atividades práticas obrigatórias enfrentaram desafios para dar continuidade às aulas, tendo que utilizar de estratégias para isso. Como foi o caso também do curso de medicina que utilizaram além do podcast, o Padlet que funciona como um "mural virtual" interativo e dinâmico que ajudou a aprimorar a construção do curso de forma on-line. Aulas de semiotécnica foram hospedadas na plataforma Anchor e no Spotify, sendo reproduzidas mais de 900 vezes em oito semanas. O aplicativo móvel Kahoot também foi utilizado como estratégia para mediar a interação com a turma (Magalhães et al. 2020).

O Padlet é um recurso que proporciona aos seus usuários acesso irrestrito, consequentemente ele se torna autônomo nos seus estudos, curtindo, comentando e avaliando os materiais expostos no mural, é possível também fazer compartilhamentos 
dos murais com outras pessoas, pois, é uma ferramenta colaborativa. Esta plataforma é compatível com temáticas em formatos diversos: vídeos, fotos, links, telas compartilhadas e desenhos (Silva \& Lima, 2018). O Padlet é um ambiente de aprendizagem virtual onde é possível anexar o material de estudo, além dos podcasts e das webconferências (Magalhães et al. 2020).

Para o ensino das línguas, também precisou haver uma adaptação para dar continuidade às aulas. No estudo de Monteiro (2020) percebeu-se o uso de ferramentas como WhatsApp (para prova oral), Linkedin, Google Maps (para ensinar direções e a parte cultural das cidades), Google tradutor (para leitura de textos e produção textual). Os professores referiram usar ainda o Kahoot (jogos para consolidar aspectos gramaticais e de uso da língua) e Quizlet, além da Netflix (para filmes com legendas). Os docentes citaram ainda o uso de aplicativos móveis como o Longman English Dicionary (aplicativo de dicionário monolíngue) e o VOA learning english (aplicativo que oferta vídeos, podcasts, matérias e atividades em vários níveis de inglês).

Os estudos de Santos et al., (2019) evidenciam que o Kahoot é uma plataforma diferenciada que os docentes usam para fazer avaliações que visam avaliar os conhecimentos prévios dos alunos. Silva (2018) aborda que através do Kahoot o ato de aprender acontece de forma significativa e agradável, por ser de fácil aplicabilidade. Diniz \& Ferreira (2018) reforçam que a aprendizagem é decorrente da compreensão dos alunos, devido ao fato da ferramenta digital ser divertida, dinâmica e diferente. Mediante isso, professores e alunos são capazes de criar jogos e quizzes interativos para aperfeiçoar a absorção de conteúdos, além de criar jogos, a plataforma permite compartilhar e jogar.

Denardi et al., (2021) também evidenciaram o uso das plataformas Kahoot e Google Maps em aulas durante a pandemia, bem como o uso do Google Meet, Zoom, Bongo, Skype, Google Hangouts, Microsoft Teams, WhatsApp, Google Classroom e o uso do Screencastify, YouCute para Edição de vídeos.

Além das estratégias mais usadas por outros autores, como é o caso do Google Meet, whatsapps, YouTube, Kahoot, YouTube, zoom e Padlet, o estudo de Gomes e Carvalho (2020), evidenciou o uso dos mapas mentais, do Mentimeter, do Flashback Express e do Vidyard.

O uso da tecnologia na educação tem exigido dos professores a capacidade de reinventar sua prática docente, pois, garantem uma aula inovadora com infinitas possibilidades de entretenimento, interação, comunicação, auxiliando de forma positiva no processo de construção do conhecimento do discente. As ferramentas digitais na educação envolvem os alunos com dinamicidade e atratividade (Santos et al. 2020). Para Nogaro (2016), quando o docente inova a sua prática pedagógica, é necessário que a modificação seja proposital visando um melhor desempenho da prática educativa, para garantir que o aluno participe ativamente do processo de ensino/aprendizagem.

Outra estratégia utilizada por poucos autores foi o podcast. De Paula e Figueiró (2020) avaliaram o uso dessa ferramenta em uma instituição de ensino em Volta Redonda-RJ, e perceberam a boa aceitação por parte dos alunos, em que relataram interesse na aprendizagem por meio desse recurso no nível superior.

O uso do podcast também foi citado por Torres et al. (2021) em que implementaram o recurso em projeto de extensão para fomentar o processo ensino-aprendizagem e a divulgação de conhecimentos na área da saúde. Ao total, foram divulgados 27 episódios de podcasts com esclarecimentos sobre a pandemia e outros temas. Os episódios foram acessados, até o momento da publicação do artigo, 4.539 vezes no Brasil e no exterior.

Já em outra pesquisa e extensão, para dar continuidade, professores a alunos de uma instituição do Pará utilizaram as ferramentas Google for Education: Google Classroom, Meet Hangout, Google Drive e Google Formulários. O projeto consistiu em ministração de capitações aos professores e alunos, habilitando-os ao uso dos recursos (Dias et al., 2020). 


\section{Considerações Finais}

Após a pandemia o sistema educacional deverá rever algumas questões relacionadas ao uso dos recursos digitais para incluir no ensino, no futuro. Mesmo com as dificuldades a escola tem adquirido experiências para associar um pouco mais o ensino ao mundo tecnológico. Apesar das marcas negativas, o momento está sendo um divisor de águas no cenário educacional. Novas descobertas, novo lugar para a cultura digital, dessa vez, mais presente dentro da sala de aula.

O uso das tecnologias na escola vem permitindo a construção do conhecimento no momento da pandemia da COVID19. Os resultados obtidos com esse estudo mostram as várias possibilidades de ferramentas digitais que podem ser exploradas para contribuir com a formação e construção do conhecimento.

Foi percebido que as ferramentas do Google foram as mais utilizadas, ou por serem de fácil acesso, fácil manuseio ou devido ao acesso gratuito. A empresa expandiu ainda suas ferramentas para permitir o acesso mundial nesse momento difícil. Muitas outras ferramentas pouco conhecidas foram exploradas pelos professores, algumas de acesso pago, mas que oferecem boas funcionalidades.

Percebeu-se que as redes sociais também foram exploradas para ministração das aulas, como o Instagram e o Facebook. O Youtube também serviu ao mesmo propósito. O WhatsApp, além de ferramenta usada para comunicação entre os docentes e alunos também foi usado como recurso nas aulas.

Apesar da deficiência e falta de estrutura das escolas e dos alunos, as ferramentas digitais foram as responsáveis por dar continuidade ao ensino durante a pandemia. Infelizmente, o acesso à internet e a aparelhos digitais é uma realidade distante para muitas pessoas. A distribuição de internet desigual entre as regiões brasileiras e os diferentes estratos de renda da população, são fatores que influenciam diretamente e que prejudicaram muitos alunos.

Com tantas possibilidades com o uso das tecnologias na educação e com desafios grandiosos, a relevância de pesquisas sobre o tema pode contribuir com a visão de que é necessário investir em estrutura e capacitação dos protagonistas no tocante ao uso das ferramentas digitais em educação. Assim, sugere-se a realização de novos estudos sobre o tema.

\section{Referências}

Alves, A. L., Mota, M. F. \& Tavares, T. P. (2018). O Instagram no processo de engajamento das práticas educacionais: a dinâmica para a socialização do ensino-aprendizagem. Revista Rios Eletrônica, São Francisco, 19(1):25-43.

Amaral, P. D. F. \& Sabota, B. (2017). PowToon: análise do aplicativo web e seu potencial mediador na aprendizagem. R. Tecnol. Soc., Curitiba, 13(28):72-89.

Bardin, L. Análise de conteúdo. (4a ed.), Edições70, 2010.

Batista Neto, J. B. S. et al. (2020). Construção de tecnologias educativas como forma de educação em saúde para a prevenção da Covid-19: relato de experiência. Revista Eletrônica Acervo Saúde. 12(9).

Bueno, M. B. T., Bueno, M. M. \& Moreira, M. I. G. (2021). O uso de tecnologias digitais e mídias sociais por profissionais da saúde no período da pandemia da COVID-19. Revista Thema, 20(1).

Carneiro, L. A. et al. (2020). Uso de tecnologias no ensino superior público brasileiro em tempos de pandemia COVID-19. Research, Society and Development, $9(8)$.

Carneiro, L. A., Garcia, L. G., \& Barbosa, G. V. (2020). Uma revisão sobre aprendizagem colaborativa mediada por tecnologias. Desafios-Revista Interdisciplinar da Universidade Federal do Tocantins, 7(2): 52-62.

Classroom, G. Google for education. (2020). classroom.google.com

Da Silva, A. P. (2021). O uso dos recursos tecnológicos no processo de ensino-aprendizagem: possibilidades e desafios. Goiânia. Universidade Federal de Goiás. 50p. https://repositorio.bc.ufg.br/bitstream/ri/19912/2/TCCG\%20-\%20Pedagogia\%20-\%20Ana\%20Paula\%20da\%20Silva\%20-\%202021.pdf.

Denardi, D. A. C., Amoroginski Marcos, R. \& Stankoski, C. R. (2021). Impactos da pandemia covid-19 nas aulas de inglês. Ilha do Desterro. 74(3): 113-143.

De Paula, S. A. \& Figueiró, R. (2020). utilização da mídia podcast como prática inovadora na educação superior. Research, Society and Development, 9(12).

De Sousa, A. S., De Oliveira, G. S., Alves, L. H. (2021). A pesquisa bibliográfica: princípios e Fundamentos. Cadernos da Fucamp, 20 (43):64-83. 
Dias, C. R. S. D. et al. (2020). Formação de professores da Educação Básica para uso das ferramentas Google na educação: uma experiência extensionista em tempos de pandemia. IX Congresso Brasileiro de Informática na Educação (CBIE 2020). Anais do XXVI Workshop de Informática na Escola (WIE 2020).

Dias, E. \& Pinto, F. C. F. (2020). “A Educação e a Covid-19”. Ensaio: Avaliação e Políticas Públicas em Educação, 28(108).

Diniz, L. M. F. \& Ferreira, F. (2018). Kahoot! um relato de experiência no contexto acadêmico. In: 26º Workshop sobre Educação em Computação (WEI 2018). SBC.

GIL, A. C. Como elaborar projetos de pesquisa. Atlas, 2002.

Góes, C. B, \& Cassiano, G. (2020.) O uso das Plataformas Digitais pelas IES no contexto de afastamento social pela Covid-19. Folha de rosto: Revista de Biblioteconomia e Ciência da Informação. 6(2):107-118.

Godoi, M. et al. (2021). As práticas do ensino remoto emergencial de educação física em escolas públicas durante a pandemia de covid-19: reinvenção e desigualdade. Revista Prática Docente. 6(1).

Gomes, E. G. S. \& Carvalho, A. B. G. P. (2020). As estratégias dos docentes com o uso de tecnologias digitais no contexto pandêmico da covid-19. EM TEIA - Revista de Educação Matemática e Tecnológica Iberoamericana. 11(2).

Iftakhar, S. (2016). Google classroom: what works and how. Journal of Education and Social Sciences, 3(1):12-18.

Julião, A. L. (2020). Professores, tecnologias educativas e COVID-19: realidades e desafios em Angola. Revista Angolana de Ciências. 2(2):01-25.

Kenski, V. M. (2007). Educação e tecnologias. Papirus editora.

Lakatos, E. M., Marconi, M. A. (2003). Fundamentos de Metodologia Científica. Atlas.

Magalhães, A. J. A. et al. (2020). O Ensino da Anamnese Assistido por Tecnologias Digitais durante a Pandemia da Covid-19 no Brasil. Revista brasileira de educação médica. 44(1).

Monteiro, F. N. S. P. (2020). As tecnologias digitais no processo de ensino e aprendizagem de línguas estrangeiras - estudo de caso de escola da Rede Federal de Ensino - CE/Brasil - em contexto anterior ao da pandemia de covid-19. Research, Society and Development, 9(10).

Moreira, J. A. M, Henriques, S. \& Barros, D. (2020). Transitando de um ensino remoto emergencial para uma educação digital em rede, em tempos de pandemia. Dialogia, 34(1):351-364.

Moodle. Modular Object-Oriented Dynamic Learning Environment. [Internet]. Disponível em: https:https://docs.moodle.org/all/pt_br/Sobre_o_Moodle.

Nonato, E. R. S., Sales, M. V. S. \& Cavalcante, T. R. (2021). Cultura digital e recursos pedagógicos digitais: um panorama da docência na covid-19. Revista práxis educacional, 17(45):1-25.

Nogaro, A. \& Battestin, C. (2016). Sentidos e cotornos da inovação na educação. HOLOS, 2:357-372.

Santos, A. C., Santos, N. A. \& Santos, W. P. (2021). Tecnologias digitais e educação escolar em tempos de pandemia da Covid-19: Percepções de professores/as de Língua Portuguesa. Educação, Sociedade \& Culturas, 59(1):97-115.

Santos, V. A. et al. (2020). O uso das ferramentas digitais no ensino remoto acadêmico: desafios e oportunidades na perspectiva docente. Conedu (Congresso Nacional de Educação)

Salvador, P. T. C. O. et al. (2019). Construção de hipermídia para apoio ao ensino da sistematização da assistência de enfermagem. Revista Gaúcha de Enfermagem, 40(1).

Senhoras, E. M. (org.). (2021). Ensino remoto e a pandemia de COVID-19. Editora IOLE.

Silva, P. G. \& Lima, D. S. (2018). Padlet como ambiente virtual de aprendizagem na formação de profissionais da educação. Revista Renote, 16(1).

Silva, M. C. P. (2018). Uso do kahoot como ferramenta de avaliação e ensino-aprendizagem no ensino da membrana plasmática. Revista Eletrônica Estácio Saúde, 7(2): 6- 9 .

Sousa, S. M. R. et al. (2021). Reflections on remote learning in the pandemic. Research, Society and Development, 10(15).

Scalabrin, A. M. M. O. \& Mussato, S. (2020). Estratégias e desafios da atuação docente de uma professora no contexto da pandemia da Covid-19. Revista de Educação Matemática, 17(1):1-19.

Torres, R. M. et al. (2021). AVAS21 - Ambiente virtual de aprendizagem para o século 21: uma experiência exitosa. Brazilian Journal of Development. 7(5).

Valente, G. S. C. et al. (2020). O ensino remoto frente às exigências do contexto de pandemia: Reflexões sobre a prática docente. Research, Society and Development, $9(9)$.

Zoom. Zoom Cloud Meetings - App. 2020. https://zoom.us/pt-pt/meetings.html. 